\title{
Tangram based technology for clothing design
}

\author{
Boryana Georgieva-Gushtanova ${ }^{1 *}$ \\ ${ }^{1}$ Technical University of Sofia, Faculty of Mechanical Engineering, Department of Engineering \\ Design, Bulgaria
}

\begin{abstract}
The trends in the society regarding environment protection and more optimized production are reason for the research of the author in this direction. The technology of the item connect with the stamina of the product. Within the report, there is an author's development for series of clothing presented in the context of the steady design. Using zero-waste technology aims the improvement of the functional characteristics, as it is being experiment with different textile materials like artificial leather, cotton, wool, spandex and others. The design through transformation of square into other figure through cutting connect with the Chinese tangram game. Problems are included, such as construction, functional, and aesthetic character, as well social and ecological aspect.
\end{abstract}

\section{Introduction}

Eco fashion is a fairly small segment of the fashion industry at present, but it is among the fastest growing ones. The ethical and eco fashion are fashion trends dealing with the proper allocation of the resources that already have for the sake of protecting the Planet Earth. These trends also seek a more rational method of operation in the fashion industry. Design, produce and dispose of clothes have a huge impact on the environment. Their production requires toxic chemicals and leads to climate change, and the public in general does not know where and how to recycle them [1].

\subsection{Tangram}

The Tangram is a dissection puzzle consisting of seven flat polygons, called tans, which are put together to form shapes. It is reputed to have been invented in China sometime around the late 18th century and then carried over to America and Europe by trading ships shortly after. It is one of the most widely recognized dissection puzzles in the world and has been used for various purposes including amusement, art, and education.

The objective is to replicate a pattern (given only an outline) generally found in a puzzle book using all seven pieces without overlap. Alternatively the tans can be used to create original minimalist designs that are either appreciated for their inherent aesthetic merits or as the basis for challenging others to replicate its outline.

* Corresponding author: info.bobbyhobby@gmail.com 
This article discusses the possibilities for zero waste clothing design by applying the principle of the Tangram [2].

\subsection{Tangram elements}

The seven polygons (Fig. 1) are: 2 large right triangles (hypotenuse 1, sides $\sqrt{2} / 2$, area 1/4), 1 medium right triangle (hypotenuse $\sqrt{2} / 2$, sides $1 / 2$, area $1 / 8$ ), 2 small right triangles (hypotenuse $1 / 2$, sides $\sqrt{2} / 4$, area $1 / 16$ ), 1 square (sides $\sqrt{2} / 4$, area $1 / 8$ ), 1 parallelogram (sides of $1 / 2$ and $\sqrt{ } 2 / 4$, height of $1 / 4$, area $1 / 8$ ). Of these seven pieces, the parallelogram is unique in that it has no reflection symmetry but only rotational symmetry, and so its mirror image can be obtained only by flipping it over. Thus, it is the only piece that may need to be flipped when forming certain shapes.

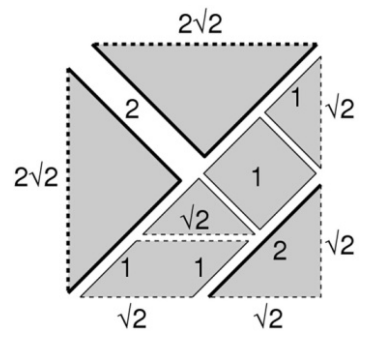

Fig. 1. Tangram seven tans.

\subsection{Zero-waste design}

The principle of full utilization of fabrics has been known ever since ancient times. Traditional kimonos in Japan are still made based on that principle because of the high cost of silk [3]. The aim is to ensure its complete use by placing the elements very sparingly on the fabric so that no waste is generated. Globally, numerous designers are working on this problem by proposing solutions that cut the fabric into either identical or different pieces along the lines of regular or irregular grids. These pieces are then assembled and creases and frills are made by using the residual pieces for decoration of the items $[4,5]$, the principles of origami [6,7], cutting the fabric into strips which are interwoven in a certain order [8], or $3 \mathrm{D}$ printing. These solutions for saving materials and achievement of zero-waste have been also successfully applied in the production of furniture [9], lighting fixtures and home textiles [10].

\section{The experiment}

The possible solution is the use of the traditional Tangram, which constitutes a square. Just one glance over the game is sufficient to notice numerous possibilities for using the proposed shapes for clothing elements. The style would be different from the style of clothing that can be achieved by traditional garment construction. The shapes used in the game are more suitable for outerwear, blouses and loose cut skirts. Constructing pants by using this method would be a particular challenge. As part of this first experiment, three pieces of clothing of the same construction will be made, however, textile of different characteristics will be used in order to change the appearance of the clothing [11]. A piece of outerwear clothing and two dresses will be made in order to study the behavior of zero waste construction when using different materials. 


\subsection{Construction}

The output shape for cutting will be a square, the side of which will be equal to the width of the roll of fabric. The selected textiles will be of the most common width of $140-150 \mathrm{~cm}$, where this range does not have any particular effect on the final product. The positioning of the elements of the cut is presented on Fig. 2. The cut follows the principle of axial symmetry along the vertical axis of the model. This principle has been selected based on logical reasoning and it is also present in traditional garment construction. The two large right triangles are placed at the two sides of the axis of symmetry, the medium right triangle is positioned symmetrically at the two sides of the axis and, in order to obtain two more symmetric details, the two small right triangles are connected so that they look like a parallelogram. In addition, a hood for the clothing is made by using the square of the Tangram.

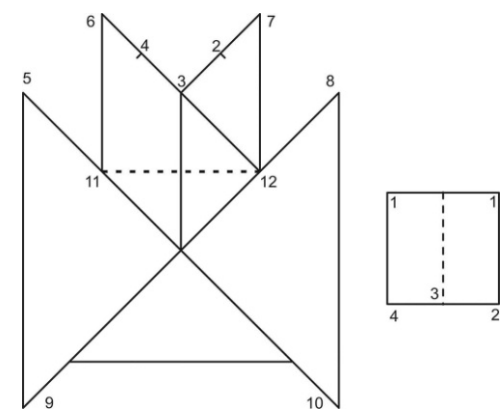

Fig. 2. The positioning of the elements of the cut.

\subsection{Variations of models}

After connection of the details a mannequin is used for this flat shape and the clothing is adjusted to the body based on the moulage technique. We attempt to vertically align lines 59 and 8-10. Diversity of the clothing front line can be achieved by using different fastening methods, such as a zipper, buttons, etc. A variation of the bust size can be achieved when joining the edges at points 5, 6, 7 and 8 by their different intertwining. Along the 11-12 line at the back (near the waist), the model can be changed by making frills with an elastic band, a cord, etc. Different types and sizes of pleats and peplums can be made.

\subsection{Possibilities of using double-faced and patterned fabrics}

Depending on the choice of textile and the clearly oriented patterns of the fabric, such as stripes or chequered pattern, a large variety of designs may be obtained by focusing on the detail orientation. Another possibility for development of a different design is the selection of double-faced fabric. Models made of it can be worn on either side and the faces of the details can also be interchanged, as shown on the drawing on Fig. 3. These two possibilities can be seen to some extent in Model 1 (Fig.4). Additional variety may be obtained from the method of connection of the details - by overlapping, lock stitch or decorative stitches. They can be connected with different types of braids and bias strips or colored threads. The choice of strips and threads in contrasting colors will put emphasis on the innovative construction. 

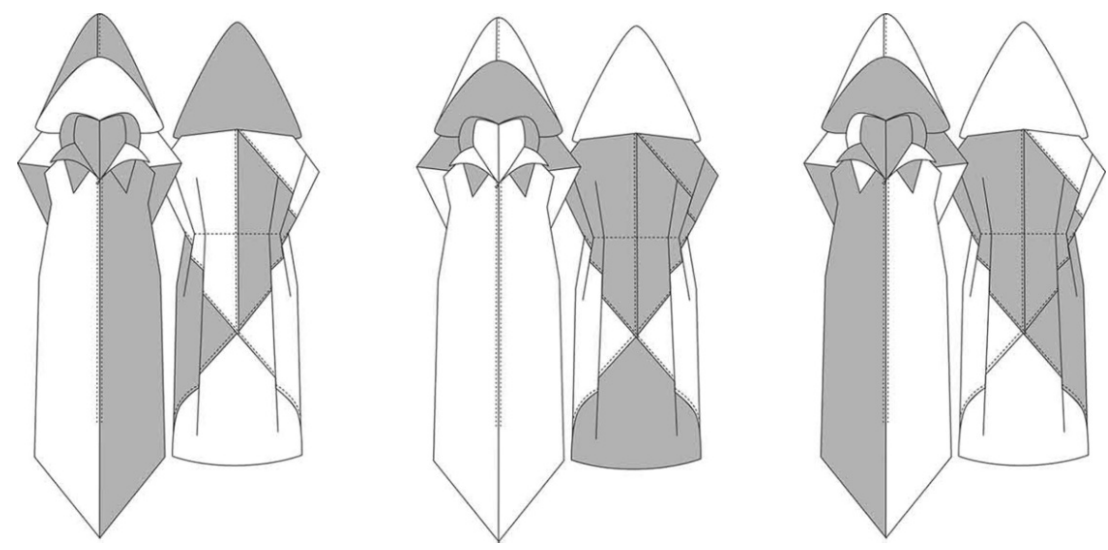

Fig. 2. Interchanged details - more different visions of the same cut on Model 1.

\section{One cut behind three different garments}

\subsection{Model 1}

For the experiment with Model 1 (Fig. 4), which is a vest made of fabric for upholstery, twill weave (composition $100 \%$ polyester), which makes it look like a striped model. The model has a large hood. When the hood is on the head, it takes the shape of a triangle and gently falls on the shoulders. When it is taken off, its top reaches the waist. The edges of the details are finished with overlock machine and are connected with a red thread through a zigzag stitch. The intertwining of the corners at points 5, 6, 7 and 8 forms an interesting detail that looks like a lapel. The model is sleeveless, with covered shoulders, and it is fastened with a zipper. Its length is mid-calf and the corners of the large triangles at the front reach the ankle. The back of the model is fitted along the 11-12 line by using four peplums positioned symmetrically - two on each side.

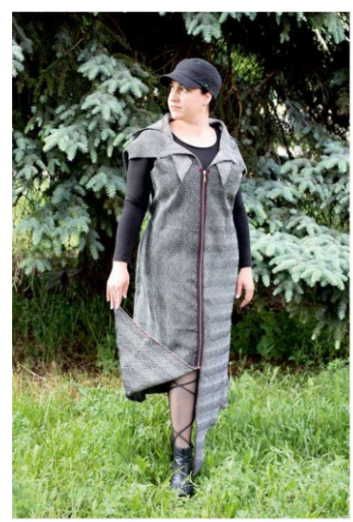

a

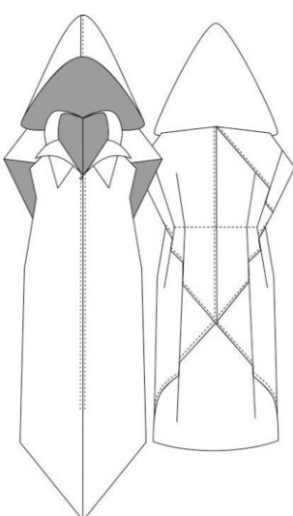

b

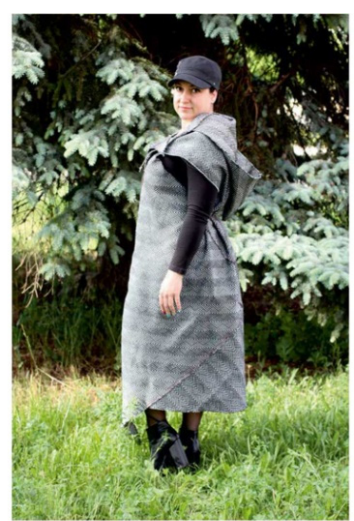

C

Fig. 4. Model 1: a) Front view with detail that looks like a lapel; b) Flat sketch; c) Large hood and peplums. 


\subsection{Model 2}

For the experiment with Model 2 (Fig.5), dual layer BlackOut fabric with woven black yarn (textile for curtains, composition $100 \%$ polyester) has been used. This is a heavy weight fabric which forms soft creases in the presence of frills. The model constitutes a calf length sleeveless dress. The dress is sewn at the front and no fastening has been designed. The entire dress is decorated with frills with ornamental and functional purpose. The details are connected with lock stitch finished with overlock machine. The frills are made by using threaded cords with stops. The frills along the neckline allow some adjustment of the bust size. The waist circumference can be adjusted with a cord threaded along the 11-12 line (Fig. 2 ). There is a threaded cord along the edge and the central line of the hood, which allow transformation of the hood into a generously pleated collar. A cord threaded along the lower hem of the skirt completely changes the silhouette. The cord channels are made by folding the edges of the details, whereas the ones along the 11-12 line is made using a bias strip.

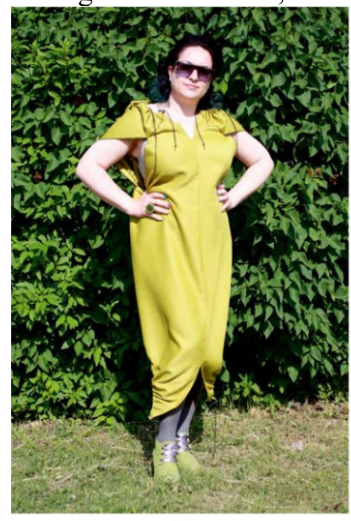

a

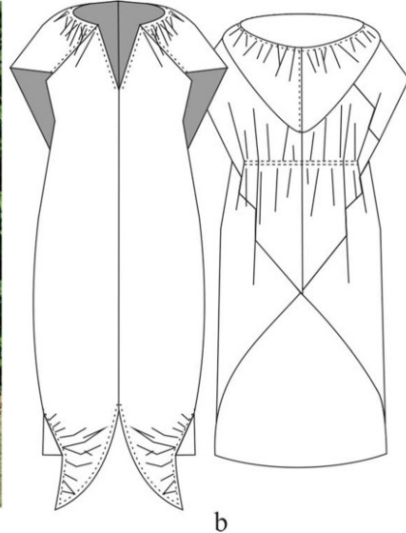

b

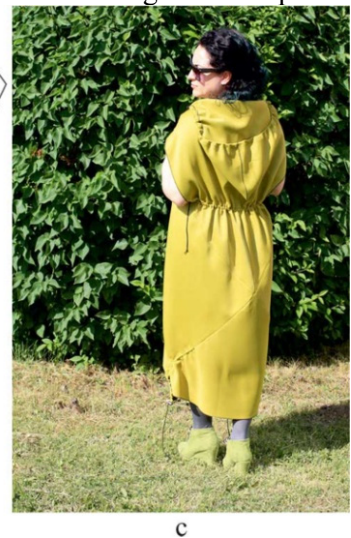

C

Fig. 5. Model 2: a) Front view; b)Flat sketch; c) Back view

\subsection{Model 3}

Spandex has been used for the experiment with Model 3 (Fig. 6). It is a mid-calf sleeveless sports dress. The details of the model are connected by overlapping, as in Model 1. This leaves the stitches visible and they serve as an additional engaging element in the design because of the property of the spandex to roll at the edges. The details do not need finishing and the edges are left as they are. They can be decorated with ornamental stitches. No fastening has been designed and the dress is closed with a front seam from the neckline to the knees. The corners of the triangles at the neckline fall freely and can be tied in different combinations of knots. The hood has a frill along the edge made by sewing an elastic band. The same frill has been made along the 11-12 line (Fig. 2). The corners of the large triangles which reach the ankle at the front side can also be modified by tying different knots. 


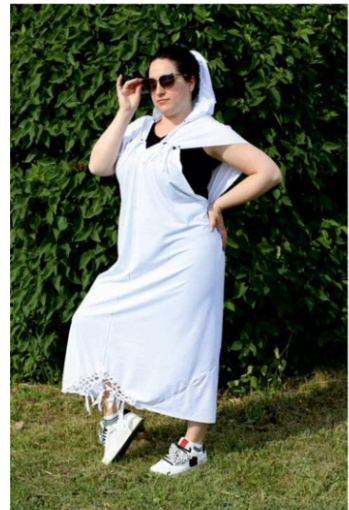

a

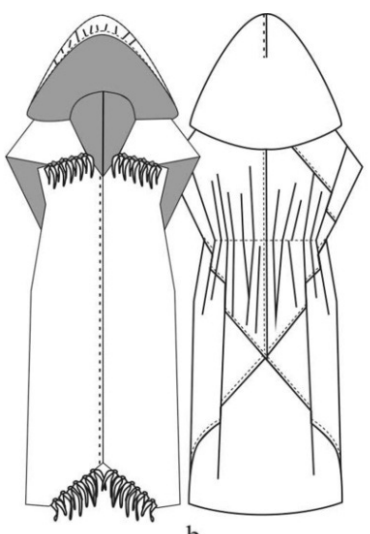

b

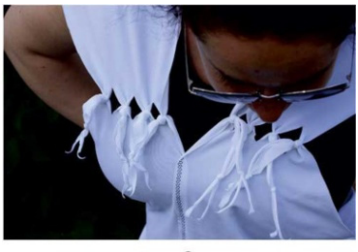

$\mathrm{c}$

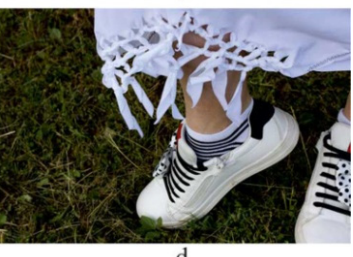

d

Fig. 6. Model 3: a) Finished model with hood; b) Flat sketch; c) Knots on the neck; d) Knots on the front side.

\section{Conclusion}

The items are suitable for people of different height and weight, however, they need to be customized based on the moulage technique. The length of the entire item from the shoulder to the edges of the triangles at the front is $120 \mathrm{~cm}$ and the length of the back is $100 \mathrm{~cm}$. If the corners on the front side in Model 1 make movement difficult, they can be sewn and folded in any direction, which would reduce the length of the item. For the dresses, this length can be adjusted by the frills and knots. The waist circumference of the item, without the frills, is $130 \mathrm{~cm}$, and the openings for the arms are about $70 \mathrm{~cm}$.

The additional materials used for all three models are bias strips, elastic bands, cords, caps and stops. These are materials that are nearly $100 \%$ utilized and can be adopted by the zerowaste clothing production technology.

Given that the entire width of the roll of fabric is used, its end is cut in a straight line and the additional materials are offered per linear meter, therefore the goal is achieved and the proposed design is implemented zero-waste.

The fact that the clothes allow them to adapt to a wider range of bodies makes them a sustainable design.

Despite the great possibility for use by people of different build and height, this type of design may be relatively difficult to adopt in certain spheres, for examples the ones that require very formal style. However, the future of the planet and our willingness to conserve it suggests that innovative methods for clothing production will be increasingly adopted and the new look will be accepted more widely.

\section{References}

1. D. Thomas, Fashionopolis: Why What We Wear Matters, Penguin Books, ISBN: 978073-522-403-2, (2020)

2. S. Loyd, The Book of Tangrams: 700 Puzzles, Dover Publications, ISBN: 978-048-683386-6, (2019)

3. K. Nitanai, Kimono Design: An Introduction to Textiles and Patterns, Tuttle Publishing, ISBN: 978-480-531-428-9, (2017) 
4. R. Atanasova, Women's clothing with eco design features, E3S Web of Conferences, 207, 03005, (2020)

5. T. Rissanen, H. McQuillan, Zero Waste Fashion Design, Bloomsbury Visual Arts, ISBN: 978-135-009-483-3, (2018)

6. B. Georgieva-Gushtanova, Origami based technology for handbag production, Proceedings of the 15th International Conference Standardization, Prototypes and Quality, Turkey, ISBN: 978-975-374-247-4, 273-279, (2019)

7. https://www.isseymiyake.com/

8. https://www.behance.net/CarlosVillamil

9. G. Chervendinev, E. Ochkova-Dimitrova, Possibilities of using cad products and parametric design in the sculpture (project "torch of knowledge"), Proceedings of the Jubilee Scientific Conference, "Angel Kanchev" University of Ruse, Bugaria, ISSN: 131-133-21, 54, 237, (2015)

10. E. Ochkova-Dimitrova, The surface as form-formation factor (project „lamp“), Proceedings of the XV National Conference with International Participation on Lighting BulLigh ISSN: 131-407-87, 244, (2014)

11. M. Panchev, Using the possibility of inorganic nano solution for special gentrification, Proceedings of Scientific conference of FPEPM Technical University of Sofia, 2, 250, (2015) 